\title{
Review Article \\ Defensins: The Case for Their Use against Mycobacterial Infections
}

\author{
Haodi Dong, ${ }^{1}$ Yue Lv, ${ }^{1}$ Deming Zhao, ${ }^{1}$ Paul Barrow, ${ }^{2}$ and Xiangmei Zhou ${ }^{1}$ \\ ${ }^{1}$ National Animal Transmissible Spongiform Encephalopathy Laboratory, Key Laboratory of Animal Epidemiology \\ and Zoonosis of Ministry of Agriculture, College of Veterinary Medicine and State Key Lab of Agrobiotechnology, \\ China Agricultural University, Beijing, China \\ ${ }^{2}$ School of Veterinary Medicine, University of Nottingham, Sutton Bonington, Loughborough, Leicestershire Le12SRD, UK
}

Correspondence should be addressed to Xiangmei Zhou; zhouxm@cau.edu.cn

Received 4 July 2016; Accepted 30 August 2016

Academic Editor: Margarete D. Bagatini

Copyright (C) 2016 Haodi Dong et al. This is an open access article distributed under the Creative Commons Attribution License, which permits unrestricted use, distribution, and reproduction in any medium, provided the original work is properly cited.

Human tuberculosis remains a huge global public health problem with an estimated 1/3rd of the population being infected. Defensins are antibacterial cationic peptides produced by a number of cell types, most notably neutrophil granulocytes and epithelial cells. All three defensin types $(\alpha-, \beta$-, and $\theta$-defensins) have antibacterial activities, mainly through bacterial membrane permeabilization. Defensins are effective against Gram-negative and Gram-positive bacteria including mycobacteria and are active both intra- and extracellularly. Mycobacterial resistance has never been demonstrated although the $m p r F$ gene encoding resistance in Staphylococcus aureus is present in the Mycobacterium tuberculosis genome. In addition to their antibacterial effect, defensins are chemoattractants for macrophages and neutrophils. There are many cases for their use for therapy or prophylaxis in tuberculosis as well. In conclusion, we propose that there is considerable scope and potential for exploring their use as therapeutic/prophylactic agents and more comprehensive survey of defensins from different species and their bioactivity is timely.

\section{Introduction}

Tuberculosis remains the most important infectious disease globally, and Mycobacterium tuberculosis is thought to be present in one-third of the world's population with 8-10 million new cases of active tuberculosis occurring annually worldwide. In 2013, an estimated 9.0 million people developed tuberculosis, and 1.5 million died from the disease [1]. Almost all cases of tuberculosis are caused by M. tuberculosis, with $M$. bovis contributing less than $1.4 \%$ of all pulmonary cases outside of Africa and $2.8 \%$ of cases in Africa with a crude incidence of 7 cases per 100,000 population [2]. The incidence of disease in some countries is also exacerbated by HIV infections [1]. Because of the difficulties of chemotherapy, the incidence of multidrug-resistant strains of $M$. tuberculosis has increased in many areas during recent decades [1]. This deterioration in the global situation highlights the need for new therapeutic agents against mycobacterial diseases. Recent research has shown that defensins have bactericidal activity against $M$. tuberculosis and indicate their potential to play a more significant role in tuberculosis control than what was previously considered [3].

Protective immunity against mycobacterial infections requires the generation of an effective cell-mediated immunity [4]. However, an efficient innate immune response may also be important in natural resistance against mycobacterial infection in addition to maintaining longer-term control of bacillary growth during latent infection. Alveolar macrophages and lung epithelial cells are the main cells to first encounter $M$. tuberculosis during primary infection. Studies of human airway epithelia in vivo or in vitro show that they can generate antimicrobial activity through the production of antimicrobial peptides $[5,6]$. In addition, defensins account for a high proportion of the antibacterial activity associated with neutrophil granules $[7,8]$. Defensins act as a bridge linking innate and acquired immunity largely through their chemotactic properties. They belong to a family of small (3$5 \mathrm{kDa}$ ) cationic cytotoxic and oxygen-independent peptides 
that are active against a wide spectrum of microorganisms including bacteria, viruses, and fungi [9]. This review presents a brief summary of their role in immunity with specific reference to human and animal tuberculosis and explores their potential as a novel approach to therapy or prophylaxis.

\section{Defensins in Man and Animals}

There are three major classes of defensins expressed by different cells within the vertebrate world; $\alpha$-, $\beta$-, and $\theta$-defensins are differentiated by their structure and antimicrobial activity.

$\alpha$-defensins, which have been identified in humans, monkeys, and several rodent species, are particularly abundant in polymorphonuclear neutrophils (PMNs), certain macrophage populations, and Paneth cells of the small intestine [8, $10,11]$. Human neutrophil $\alpha$-defensins (HNPs) $1-4$ constitute $\sim 30 \%$ of proteins in the azurophilic granules of PMNs [8]. However, against Gram-positive bacteria, HNP-1, HNP-2, and HNP-3 account for most of the total defensin content and have greater antimicrobial activity than HNP-4 [12]. In contrast, the potency of HNP-4 against Gram-negative bacteria is greater than those of HNP-1 and HNP-2 [13]. $\alpha$ Defensins 5 and 6 are found in Paneth cells, the epithelial granulocytes of the small intestine $[14,15] . \alpha$-Defensins are also found in alveolar macrophages from rabbits but not from humans [16]. $\alpha$-Defensins show activity in vitro against Gram-negative and Gram-positive bacteria and fungi [12] that can be modulated by environmental conditions such as redox and $\mathrm{pH}[17]$.

In contrast to $\alpha$-defensins, $\beta$-defensins are largely expressed in epithelial tissues $[18,19]$. The first $\beta$-defensin was identified in bovine tongue tissue, and $\beta$-defensins subfamilies have now been reported in primates (humans and apes), bovines, and rodents such as rats and mice [6, 20]. Furthermore, pigs and other farm animals including birds express only $\beta$-defensins [20-22]. As the most comprehensively studied, $\beta$-defensins possess the widest taxonomic distribution, including invertebrates and plants, indicating an ancient point of origin [23]. Human $\beta$-defensin 1 (HBD1 ) is expressed constitutively by a number of body systems including the urogenital tract and respiratory tract $[24,25]$. HBD-2 was discovered in extracts of lesional scales from patients suffering from psoriasis [26] and is expressed by inflamed skin, lung, oral mucosa, and ocular surfaces [27]. Its expression by epithelial cells can be induced by TNF- $\alpha$ [28] and interleukin- (IL-) $1 \beta$ but also by bacteria [18]. HBD-3 is expressed mainly by the skin and tonsils [29], and HBD-4 is expressed by many tissues but is particularly highly expressed in the gastric antrum and testes [30]. The expression of HBD1 is constitutive, whereas HBD-2-4 are inducible [31] and are thought to play a crucial role against bacterial infection as part of the epithelial barrier [32]. Proinflammatory cytokines, bacteria, and fungi have all been found to increase the expression of these defensins in cultured keratinocytes [20].

$\theta$-Defensins are the only defensin subfamily with a circular structure, which likely originated through mutation of a preexisting $\alpha$-defensin gene in Old World monkeys [33]. They were first found in the monocytes and neutrophils of the rhesus monkey (Macaca mulatta) and are the least numerous subfamily of mammalian defensins [34], with only three $\theta$ defensins recognized (rhesus theta defensin (RTD) 1-3) from studies within leukocytes [34]. Circular defensin isoforms also exist with five identified in peripheral blood leukocytes and four in the bone marrow in the olive baboon (Papio anubis). Less is known about the distribution and diversity of $\theta$-defensins because only 11 different $\theta$-defensins have been isolated from three species of primates since their discovery in 1999 [35]. The antimicrobial activity of $\theta$-defensins is thought to be their natural function [35], and they bind to and neutralize bacterial toxins $[36,37]$. For example, the human $\theta$-defensin retrocyclin- 1 and its analogues are active against C. albicans and L. monocytogenes and bind to anthrax lethal toxin [37].

To date, more than 17 human defensins have been reported [10]. However, many more $\beta$-defensins have been reported by in silico analysis [38], and a genome-wide computational search has identified more than 30 human uncharacterized $\beta$-defensin genes with full biological significances currently undetermined [39].

\section{Mechanism of Action; the Role of Defensins in Immunity}

3.1. Chemotaxis. The role of defensin chemotactic activity in initiating and regulating the immune response is now well known [30-32]. The human $\alpha$-defensin family chemoattracts macrophages [8]. Amongst $\beta$-defensins, they possess chemotactic activities for immature memory T-cells and dendritic cells through the chemokine receptor CCR6 [40]. HBD-2 and HBD-3 can combine with both CpG and host DNA to form aggregates that resemble DNA nets, which may enhance the intracellular uptake of $\mathrm{CpG}$ and self DNA and activate plasmacytoid dendritic cells (pDCs) to promote DNA-induced IFN- $\alpha$ production in a Toll-like receptor 9- (TLR9-) dependent manner [41]. Subcutaneous injections of these complexes showed enhanced infiltration of inflammatory cells at the injection site, indicating a potential pathophysiological role for defensin/DNA complexes in contributing to inflammation [41]. A recent study showed that murine $\beta$-defensin 2 (mBD-2) immunostaining in tuberculous mice was essentially localized to cells with dendritic morphology located near the mediastinal lymph nodes and showed a high level of gene expression [42]. This suggests that $\beta$-defensins may play an important role in the initiation of a Thl response as a link between the innate and adaptive immune responses [42]. HNPs can also promote B- and T-cell interactions by modulating the Th1- and Th2-type cytokines $[43,44] . \theta$-Defensins have not been found to possess chemotactic activity.

The expression of HBD-2 by human macrophages can be triggered by $M$. tuberculosis [45]. The expression and production of defensins are activated by a number of routes including direct recognition of pathogen-associated molecular patterns (PAMPs), such as LPS, by TLR [46]. This initiates MAPK- or NF- $\kappa \mathrm{B}$-dependent cascades that culminate in a proinflammatory response involving the secretion of cytokines, chemokines, and defensins [19], which themselves also have the capacity to induce defensin secretion. Their 
expression is also mediated by receptors other than TLRs, including NOD2 [47]. Thus, infection of cells with live Mycobacteria leads to the induction of TNF $\alpha$ and HBD-2 [48].

Defensins are also thought to contribute to the inflammatory processes by inducing histamine release by mast cells and increasing hyperresponsiveness of the airways to histamine [49]. Besides their antimicrobial and potential proinflammatory activities, defensins also display anti-inflammatory roles by binding to $\mathrm{Clq}$ to inhibit activation of the classical pathway of complement activation [49] and have been demonstrated to inhibit fibrinolysis [50]. Some defensins are also able to limit the inhibitory action of glucocorticoids on the suppressor functions of T-lymphocytes, which was abolished after adrenalectomy [51].

3.2. Antibacterial Activity. The main mode of antibacterial action is the direct lysis of microorganisms through permeabilization of cell membranes [8] either whilst the bacteria are extracellular or after phagocytosis [52]. It is believed that electrostatic binding between the arginine groups of cationic defensins and membranes rich in anionic phospholipids induces the formation of voltage-regulated channels, which causes the leakage of intracellular metabolites [8]. Some defensins can also bind avidly to membrane glycoproteins [53], which may be in part responsible for their antiviral activity. This is supported by the fact that defensins do not have antiviral activity against nonenveloped viruses [8].

In addition, defensins can bind to polyanionic molecules such as DNA via electrostatic interaction after entering the bacterial cell [54]. Since HNPs also target genomic DNA, inducing single-strand DNA breaks [55], it has been hypothesized that adenosine $5^{\prime}$-diphosphate ribosylation might play a regulatory role in the biological properties of arginine-rich HNPs [56]. Defensins are highly antibacterial even in micromolar concentrations for both Gram-negative and Grampositive bacteria, including mycobacteria. Unfortunately, the relative antibacterial activity between $\alpha$ - and $\beta$-defensins is still unknown.

3.3. Bacterial Resistance to Defensins and Cytotoxicity. Pathogens that colonize sites where defensins may be present in high concentrations have developed mechanisms to resist their antibacterial activity. A number of genes responsible for defensin resistance have been identified in different pathogens. A staphylococcal gene, $m p r F$, confers resistance to several antimicrobial peptides including defensins and related genes and has been found in the genomes of several other pathogens such as M. tuberculosis, Pseudomonas aeruginosa, and Enterococcus faecalis [57]. MprF reduces the negative charge of the membrane surface and leads to decreased binding of the cationic defensins by modifying phosphatidylglycerol with L-lysine in the membrane lipids [57]. The phoP gene in Salmonella enterica serovar Typhimurium is also thought to contribute to defensin resistance because a phoP mutant shows significantly greater sensitivity to defensins [58].

Potential clinical application of defensins must thus be reviewed in the light of possible development or acquisition of resistance. However, their nonspecific mode of action suggests that they should show promise in averting the development of resistance. Moreover, studies have demonstrated that resistance is less frequent than that observed for conventional antibiotics [59-61], and selections for resistance in susceptible strains of $M$. tuberculosis, Pseudomonas aeruginosa, and Enterococcus faecalis have failed [62].

In addition to their toxic effects on microorganisms, there are a number of reports indicating cytotoxicity for eukaryotic cells. High concentrations of HNPs are found in the airway secretions of patients with chronic inflammatory lung disorders. It has been reported that HNPs are cytotoxic to airway epithelial cells and can induce chemokine secretion in several cell types such as macrophages [63]. Other studies showed that HNPs are cytotoxic not only against various kinds of human and murine tumor cells but also against a wide range of normal cells, including human endothelial cells, lymphocytes, murine thymocytes, PMNs, and spleen cells in a concentration-dependent $(25-100 \mu \mathrm{g} / \mathrm{mL})$ and timedependent manner $[62,64]$. As cytotoxic molecules, HNPs can cooperate with hydrogen peroxide, which is also secreted by activated neutrophils, to affect synergistic cytolytic activity in vitro. This interaction may contribute to granulocyteinduced cytotoxicity in vivo $[59,65]$.

Some studies showed that the minimum inhibitory concentration and median inhibitory concentration of HNP1 were $2.5 \mu \mathrm{g} / \mathrm{mL}$ and $0.8 \mu \mathrm{g} / \mathrm{mL}$, respectively, which are much lower than the harmful concentrations to normal cells [60]. This indicates that defensins have a relatively low level of cytotoxicity to normal cells at antimicrobicidal concentrations. Low cytotoxicity in vitro might be due to the presence of fetal bovine serum in the culture media, as serum proteins can protect mammalian target cells [61]. The inhibitory effect of fetal calf serum (FCS) on lysis and binding can be completely accounted for by its content of albumin [61]. Not only could FCS prevent defensin binding, but also it removed membrane-bound defensin molecules from the targets [61]. Several proteins that can bind to defensins have been identified, and some of them may work as defensin carriers for clearance from tissues and blood [32].

\section{Antimycobacterial Activity}

A high concentration of $\alpha$-defensins has been detected in bronchoalveolar lavage (BAL) samples and pleural fluid from patients suffering from pulmonary tuberculosis, and significant levels of $\beta$-defensins have been detected in bronchoalveolar lavage fluid from patients with $M$. avium-intracellulare infection [66]. Moreover, studies of gene expression profiles by microarray of peripheral blood mononuclear cells (PBMC) from patients suffering from tuberculosis and $M$. tuberculosis-infected healthy individuals who had repeated close contact to tuberculosis patients (such as a nurse, physician, or family member) and were tuberculin skin test positive showed that the concentrations of effector molecules, $\alpha$ defensins 1,3 , and 4 , were upregulated in the diseased patients [67]. Furthermore, mice infected with $1.5 \times 10^{4} \mathrm{CFU}$ of $M$. tuberculosis $\mathrm{H} 37 \mathrm{Rv}$ and treated with different doses of HNP1 injected subcutaneously showed significantly improved 
clearance of bacilli from the lungs, liver, and spleen [68]. HNP-1 at $5 \mu \mathrm{g} / \mathrm{mL}$ killed $M$. avium-intracellulare in vitro at the optimal $\mathrm{pH}$ for bactericidal activity $(>5)$ [69]. The minimal inhibitory concentration (MIC) of HNP-1 against M. tuberculosis in vitro in one study was $2.5 \mu \mathrm{g} / \mathrm{mL}$ [60], much lower than that reported in a second study $(25 \mu \mathrm{g} / \mathrm{mL})$ [70]. The difference in MIC observed could be attributed to differences in experimental design.

HNP was the first defensin found to be effective against nontuberculous mycobacteria, including $M$. avium-intracellulare [69]. HNP-2 and HNP-3 were as effective in killing M. avium-intracellulare in vitro as HNP-1 [69]. For HNP-5, the linear peptide derived from its $\mathrm{N}$-terminal fatty acylation can enhance activity against $M$. tuberculosis almost comparable to the native peptide [71].

Bovine and rabbit defensins have similar or more potent antimycobacterial activity than HNPs, especially against $M$. tuberculosis clinical isolates in vitro $[72,73]$. In contrast, another study showed that HNP-1-3 are not necessarily more effective in killing $M$. tuberculosis even at a much higher concentration, which may be the result of differing levels of resistance in the individual strains (M. tuberculosis Erdman) used [74]. Defensins may play a much more significant role in immunity against mycobacteria than what was previously thought. One can therefore speculate that defensin production, especially by epithelial cells and neutrophils, is likely to be more important early in infection before the establishment of the granuloma.

Based on its antimicrobial activity and function in host immunity, neutrophil-macrophage cooperation against $M$. tuberculosis may thus be involved in clearance [75] based on the following rationale. First, HNPs clearly show antimicrobial activity against $M$. tuberculosis in vitro by increasing the permeability of the mycobacterial cell envelope [76]. Second, in addition to direct antimicrobial activity, HNPs secreted by neutrophils recruited into the early lesion are clearly able to act as chemotactic factors, attracting immune cells including macrophages, T-lymphocytes, mast cells, and immature memory T-cells [8]. Third, HNPs released by neutrophils recruited in the early lesion can also modulate cytokine production to influence the inflammatory response since TNF- $\alpha$ secreted by macrophages may stimulate neutrophil mycobactericidal activity, which might be mediated simultaneously by defensins [74].

In vitro, the capacity of macrophages to control $M$. tuberculosis growth is improved by transfecting human monocytederived macrophages with the HBD-2 gene compared with non-HBD-2-transfected cells [77]. HBD-2 [78] shows great antimicrobial activity against $M$. tuberculosis $\mathrm{H} 37 \mathrm{Rv}$ in vitro [78]. Like HBD-2, HBD-1 also plays a role in immunity against $M$. tuberculosis by permeabilization of both the mycobacterial cell wall and the cell membrane [79]. M. tuberculosis infection of endothelial cells in vitro also results in HBD-1 overexpression and profound cytoskeletal rearrangement [80]. One study found that infection of human limbocorneal fibroblasts with M. tuberculosis, M. abscessus, and M. smegmatis results in overexpression of HBD-1-3 [48]. Recent studies have emphasized the role of HBD-4, which can be triggered through the IL- $1 \beta$ and vitamin $\mathrm{D}$ receptor (VDR) pathways in the innate immune defense against $M$. tuberculosis survival in infected macrophages [81]. One study showed that intratracheal administration of L-isoleucine into mice infected with the antibiotic-sensitive strain H37Rv or a multidrug-resistant clinical isolate can significantly upregulate $\beta$-defensins 3 and 4 and decrease bacillary loads by inducing their gene expression [82], demonstrating that it may be possible to use defensins for treating infection by modulating their gene expression.

Interestingly, more highly virulent $M$. bovis strains induce lower levels of murine $\beta$-defensin 4 (mBD4) expression than strains of lower virulence during many time points of early infection [83], indicating the ability to suppress induction early in infection in vivo. In experimental tuberculosis, expression of $\mathrm{mBD} 3$ and $\mathrm{mBD} 4$ by airway epithelial cells in the early stages of infection correlated with temporary control of mycobacterial growth [84]. Similarly, high and stable production $\mathrm{mBD} 4$ during latent infection is associated with longterm control of mycobacterial proliferation [83]. The activity of defensins against mycobacteria coupled with their induction by infection suggests that the introduction of defensins either prophylactically or early in infection may affect the course of the disease to the benefit of the host. There thus exists the potential for the use of defensins as new prophylactic/therapeutic agents against mycobacterial infections.

\section{Antimycobacterial Therapy}

The effective in vitro activity of a number of defensins against mycobacteria combined with their beneficial chemotactic effects suggests that therapeutic or prophylactic administration of defensins or their induction in the body might lead to improvement in the course of infection and host health.

To date, there are relatively few reports on the effects of defensin administration against $M$. tuberculosis, but most of these show beneficial effects. Mice transfected with the $\beta$ defensin 2 gene showed higher survival and lower bacterial burden after challenge [85]. HNP-1 injected postinfection showed significant time- and dose-dependent clearance of bacilli from lungs, livers, and spleens in mice experimentally infected with M. tuberculosis H37Rv [68]. The HNP-1 administered to mice in that study was significantly less ( 1 and $5 \mu \mathrm{g}$ per mouse) than the concentration required $(50 \mu \mathrm{g} / \mathrm{mL})$ for antimycobacterial activity in vitro. The higher in vivo potency of HNPs is likely due to their immune enhancing effects, such as chemotaxis of T-cells [86] and monocytes [87].

Human defensins are reported to show synergistic activity with antituberculosis drugs, which suggests that they may be a promising adjunct to antituberculosis chemotherapy [88]. A number of studies have explored the combined effect of defensins and antituberculosis drugs against intracellular mycobacteria. In vitro studies suggest that HBD-2 is involved in reducing $M$. tuberculosis growth, and the combination of HNP-1 with antituberculosis drugs (i.e., isoniazid and rifampicin) resulted in a significant reduction $(P<0.001)$ in mycobacterial load [76]. HBD-1 has a lower activity against M. tuberculosis, and its combination with isoniazid significantly reduced $M$. tuberculosis growth in comparison with 


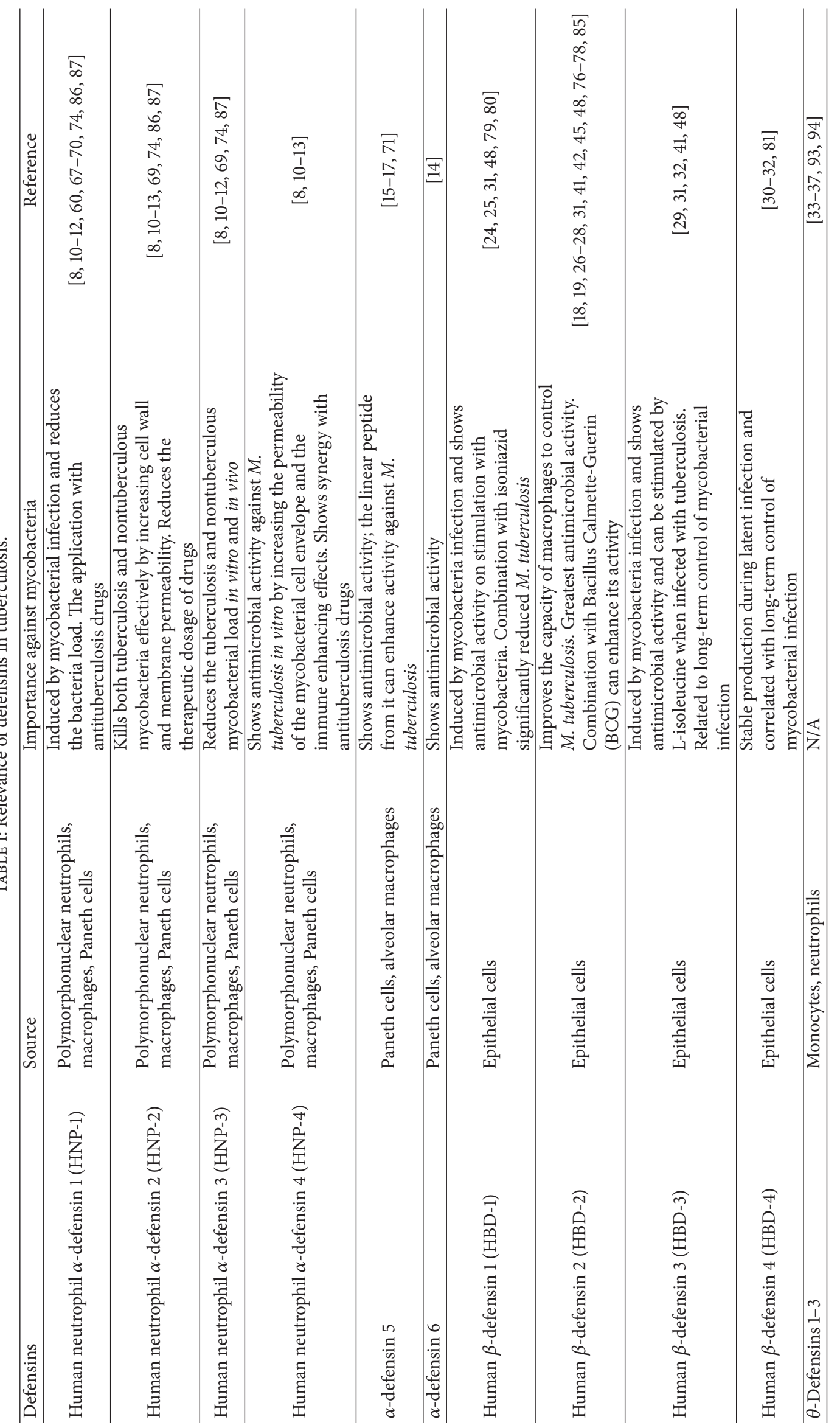


the peptides or isoniazid alone by permeabilization of both the mycobacterial cell wall and the cell membrane [79]. Moreover, a protective role for $\alpha$-defensin against mycobacterial infection has been reported in human eosinophils [89]. $\alpha$-Defensin released by eosinophils upon stimulation with lipomannan from $M$. bovis BCG, when used with eosinophil cationic protein, showed a synergistic inhibitory effect on mycobacterial growth inhibition [89].

Since the peculiar mycobacterial cell envelope is considered to contribute to the resistance to conventional antimycobacterial drugs, the combination of HNPs and antituberculosis drugs against $M$. tuberculosis $\mathrm{H} 37 \mathrm{Rv}$ not only results in increased permeability of both the mycobacterial cell wall and the cell membrane but also increases the access to intracellular targets for antituberculosis drugs. Therefore, antimicrobial peptides are potential adjuncts to chemotherapy together with conventional drugs against tuberculosis. Antimicrobial peptides can be more potent in vivo because of their immune enhancing effects by acting as a chemotactic factor and regulatory factor, interacting with immune cells like T-cells and monocytes and modulating the production of cytokines and inflammation.

Studies have shown that M. bovis BCG-induced HBD2 mRNA expression in human epithelial cells can influence protection against $M$. tuberculosis challenge [90], and $M$. bovis BCG cell wall components $(18-30 \mathrm{kDa})$ can stimulate human pulmonary epithelial cells to express defensins [91]. It also shows that prime-boost BCG vaccination with $\beta$ defensin 2 DNA vaccines can enhance the activity against M. tuberculosis [85]. It is known that protection conferred by BCG against tuberculosis is variable and can maintain longterm immunity [92], and defensins could be important as a component part of this protection against human tuberculosis.

Although $\theta$-defensins have antimicrobial activity against diverse pathogens $[35,93]$, especially viruses [94], there is, as yet, no evidence that $\theta$-defensins are involved in defense against mycobacterial infection. Table 1 shows a summary of different defensins and there function in antimycobacterium.

\section{Future Perspective}

Previous studies have widely demonstrated that defensins have activities against microorganisms including mycobacteria. Rivas-Santiago et al. found that vitamin D and Lisoleucine can induce the production of defensins by modulating their gene expression $[82,95]$. Therefore, future studies should focus on the mechanism by which defensin gene expression is modulated. On the other hand, although a large number of studies have discovered the antimycobacterial activity of defensins in vitro, there are fewer studies in vivo, and further study should include the activity of defensins against tuberculosis in vivo. Although defensins have been examined for their clinical treatment of infections with no success, defensins have a huge clinical potential, and more research into their application is needed.

\section{Conclusion}

Defensins are a family of antimicrobial peptides that are abundant amid an array of oxygen-independent antimicrobial proteins and peptides in neutrophil granules and secreted by epithelial cells. They are effective against a wide spectrum of microorganisms including mycobacteria.

The advantages and disadvantages of the various forms of defensin therapy/prophylaxis against mycobacterial infection of man and animals outlined above indicate that this could be an effective new approach to treatment and prevention of these chronic infections, which are becoming increasingly intractable to chemotherapy. Administration of defensins may have direct effects on the pathogens, stimulate innate and adaptive immunity, or be used synergistically with currently used or new chemotherapeutic agents. The experimental work with mycobacterial infections combined with their wide spectrum of activity suggests that bacterial infections other than those caused by mycobacteria may also be amenable to this approach.

\section{Competing Interests}

The authors declare that there is no conflict of interests regarding the publication of this paper.

\section{Acknowledgments}

This work was supported by the MoSTRCUK International Cooperation Project (Project no. 2013DFG32500), National Natural Science Foundation of China (Project no. 31572487), Funding of State Key Lab of Agrobiotechnology (Project no. 2012SKLAB06-14), 2015 CAU Foreign Experts Major Projects (Project no. 2012z018), and High-End Foreign Experts Recruitment Program (Project no. GDW20151100036).

\section{References}

[1] E. Dirlikov, M. Raviglione, and F. Scano, "Global tuberculosis control: toward the 2015 targets and beyond," Annals of Internal Medicine, vol. 163, no. 1, pp. 52-58, 2015.

[2] S. Singh, M. Kumar, and P. Singh, "Evolution of M. bovis BCG vaccine: is niacin production still a valid biomarker?" Tuberculosis Research and Treatment, vol. 2015, Article ID 957519, 11 pages, 2015.

[3] C. E. Rivas-Santiago, R. Hernández-Pando, and B. RivasSantiago, "Immunotherapy for pulmonary TB: antimicrobial peptides and their inducers," Immunotherapy, vol. 5, no. 10, pp. 1117-1126, 2013.

[4] J. L. Flynn and J. Chan, "Immunology of tuberculosis," Annual Review of Immunology, vol. 19, pp. 93-129, 2001.

[5] T. Ganz, "Antimicrobial polypeptides in host defense of the respiratory tract," The Journal of Clinical Investigation, vol. 109, no. 6, pp. 693-697, 2002.

[6] B. C. Schutte and P. B. McCray Jr., " $\beta$-Defensins in lung host defense," Annual Review of Physiology, vol. 64, pp. 709-748, 2002.

[7] R. Bals, "Epithelial antimicrobial peptides in host defense against infection," Respiratory Research, vol. 1, no. 3, pp. 141-150, 2000 . 
[8] R. I. Lehrer, A. K. Lichtenstein, and T. Ganz, "Defensins: antimicrobial and cytotoxic peptides of mammalian cells," Annual Review of Immunology, vol. 11, pp. 105-128, 1993.

[9] L. M. Fu, "The potential of human neutrophil peptides in tuberculosis therapy," International Journal of Tuberculosis and Lung Disease, vol. 7, no. 11, pp. 1027-1032, 2003.

[10] H. Chen, Z. Xu, L. Peng et al., "Recent advances in the research and development of human defensins," Peptides, vol. 27, no. 4, pp. 931-940, 2006.

[11] J. E. Gabay, R. W. Scott, D. Campanelli et al., "Antibiotic proteins of human polymorphonuclear leukocytes," Proceedings of the National Academy of Sciences of the United States of America, vol. 86, no. 14, pp. 5610-5614, 1989.

[12] B. Ericksen, Z. Wu, W. Lu, and R. I. Lehrer, "Antibacterial activity and specificity of the six human $\alpha$-defensins," Antimicrobial Agents and Chemotherapy, vol. 49, no. 1, pp. 269-275, 2005.

[13] C. G. Wilde, J. E. Griffith, M. N. Marra, J. L. Snable, and R. W. Scott, "Purification and characterization of human neutrophil peptide 4, a novel member of the defensin family," The Journal of Biological Chemistry, vol. 264, no. 19, pp. 11200-11203, 1989.

[14] D. E. Jones and C. L. Bevins, "Defensin-6 mRNA in human Paneth cells: implications for antimicrobia peptides in host defense of the human bowel," FEBS Letters, vol. 315, no. 2, pp. 187-192, 1993.

[15] E. M. Porter, L. Liu, A. Oren, P. A. Anton, and T. Ganz, "Localization of human intestinal defensin 5 in Paneth cell granules," Infection and Immunity, vol. 65, no. 6, pp. 2389-2395, 1997.

[16] K. A. Daher, R. I. Lehrer, T. Ganz, and M. Kronenberg, "Isolation and characterization of human defensin cDNA clones," Proceedings of the National Academy of Sciences of the United States of America, vol. 85, no. 19, pp. 7327-7331, 1988.

[17] E. M. Porter, E. Van Dam, E. V. Valore, and T. Ganz, "Broadspectrum antimicrobial activity of human intestinal defensin 5 ," Infection and Immunity, vol. 65, no. 6, pp. 2396-2401, 1997.

[18] J. Harder, U. Meyer-Hoffert, L. M. Teran et al., "Mucoid Pseudomonas aeruginosa, TNF- $\alpha$, and IL-1 $\beta$, but not IL-6, induce human beta-defensin-2 in respiratory epithelia," American Journal of Respiratory Cell and Molecular Biology, vol. 22, no. 6, pp. 714-721, 2000.

[19] P. Méndez-Samperio, E. Miranda, and A. Trejo, "Mycobacterium bovis Bacillus Calmette-Guérin (BCG) stimulates human $\beta$-defensin-2 gene transcription in human epithelial cells," Cellular Immunology, vol. 239, no. 1, pp. 61-66, 2006.

[20] J. Jarczak, E. M. Kościuczuk, P. Lisowski et al., "Defensins: natural component of human innate immunity," Human Immunology, vol. 74, no. 9, pp. 1069-1079, 2013.

[21] X. Yang, Y.-T. Cheng, M.-F. Tan et al., "Overexpression of porcine beta-defensin 2 enhances resistance to Actinobacillus pleuropneumoniae infection in pigs," Infection and Immunity, vol. 83, no. 7, pp. 2836-2843, 2015.

[22] Y. Yoshimura, "Avian $\beta$-defensins expression for the innate immune system in hen reproductive organs," Poultry Science, vol. 94, no. 4, pp. 804-809, 2015.

[23] K. G. Meade, P. Cormican, F. Narciandi, A. Lloyd, and C. O’Farrelly, "Bovine $\beta$-defensin gene family: opportunities to improve animal health?” Physiological Genomics, vol. 46, no. 1, pp. 17-28, 2014.

[24] E. V. Valore, C. H. Park, A. J. Quayle, K. R. Wiles, P. B. McCray Jr., and T. Ganz, "Human beta-defensin-1: an antimicrobial peptide of urogenital tissues," The Journal of Clinical Investigation, vol. 101, no. 8, pp. 1633-1642, 1998.
[25] M. J. Goldman, G. M. Anderson, E. D. Stolzenberg, U. P. Kari, M. Zasloff, and J. M. Wilson, "Human $\beta$-defensin-1 is a saltsensitive antibiotic in lung that is inactivated in cystic fibrosis," Cell, vol. 88, no. 4, pp. 553-560, 1997.

[26] J. Harder, J. Bartels, E. Christophers, and J.-M. Schroder, "A peptide antibiotic from human skin," Nature, vol. 387, no. 6636, p. $861,1997$.

[27] N. A. McNamara, R. Van, O. S. Tuchin, and S. M. J. Fleiszig, "Ocular surface epithelia express mRNA for human beta defensin-2," Experimental Eye Research, vol. 69, no. 5, pp. 483-490, 1999.

[28] Y. Tsutsumi-Ishii and I. Nagaoka, "Modulation of human betadefensin-2 transcription in pulmonary epithelial cells by lipopolysaccharide-stimulated mononuclear phagocytes via proinflammatory cytokine production," The Journal of Immunology, vol. 170, no. 8, pp. 4226-4236, 2003.

[29] J. Harder, J. Bartels, E. Christophers, and J.-M. Schröder, "Isolation and characterization of human $\beta$-defensin-3, a novel human inducible peptide antibiotic," The Journal of Biological Chemistry, vol. 276, no. 8, pp. 5707-5713, 2001.

[30] J. R. García, A. Krause, S. Schulz et al., "Human beta-defensin 4: a novel inducible peptide with a specific salt-sensitive spectrum of antimicrobial activity," The FASEB Journal, vol. 15, no. 10, pp. 1819-1821, 2001.

[31] T. Ganz, "Defensins: antimicrobial peptides of innate immunity," Nature Reviews Immunology, vol. 3, no. 9, pp. 710-720, 2003.

[32] T. Ganz and R. I. Lehrer, "Defensins," Pharmacology and Therapeutics, vol. 66, no. 2, pp. 191-205, 1995.

[33] P. Tongaonkar, K. K. Trinh, J. B. Schaal et al., "Rhesus macaque $\theta$-defensin RTD-1 inhibits proinflammatory cytokine secretion and gene expression by inhibiting the activation of NF- $\kappa \mathrm{B}$ and MAPK pathways," Journal of Leukocyte Biology, vol. 98, no. 6, pp. 1061-1070, 2015.

[34] R. I. Lehrer, A. M. Cole, and M. E. Selsted, " $\theta$-defensins: cyclic peptides with endless potential," The Journal of Biological Chemistry, vol. 287, no. 32, pp. 27014-27019, 2012.

[35] Y.-Q. Tang, J. Yuan, G. Ösapay et al., "A cyclic antimicrobial peptide produced in primate leukocytes by the ligation of two truncated $\alpha$-defensins," Science, vol. 286, no. 5439, pp. 498-502, 1999.

[36] A. Menendez and B. Brett Finlay, "Defensins in the immunology of bacterial infections," Current Opinion in Immunology, vol. 19, no. 4, pp. 385-391, 2007.

[37] A. C. Conibear and D. J. Craik, "The chemistry and biology of theta defensins," Angewandte Chemie-International Edition, vol. 53, no. 40, pp. 10613-10623, 2014.

[38] C. Y. Kao, Y. Chen, Y. H. Zhao, and R. Wu, "ORFeome-based search of airway epithelial cell-specific novel human $\beta$-defensin genes," American Journal of Respiratory Cell and Molecular Biology, vol. 29, no. 1, pp. 71-80, 2003.

[39] E. H. Mattar, H. A. Almehdar, H. A. Yacoub, V. N. Uversky, and E. M. Redwan, "Antimicrobial potentials and structural disorder of human and animal defensins," Cytokine \& Growth Factor Reviews, vol. 28, pp. 95-111, 2016.

[40] P. A. Raj and A. R. Dentino, "Current status of defensins and their role in innate and adaptive immunity," FEMS Microbiology Letters, vol. 206, no. 1, pp. 9-18, 2002.

[41] P. Tewary, G. De La Rosa, N. Sharma et al., " $\beta$-Defensin 2 and 3 promote the uptake of self or CpG DNA, enhance IFN- $\alpha$ 
production by human plasmacytoid dendritic cells, and promote inflammation," The Journal of Immunology, vol. 191, no. 2, pp. 865-874, 2013.

[42] B. Rivas-Santiago, A. Cervantes-Villagrana, E. Sada, and R. Hernández-Pando, "Expression of beta defensin 2 in experimental pulmonary tuberculosis: tentative approach for vaccine development," Archives of Medical Research, vol. 43, no. 4, pp. 324-328, 2012.

[43] Y. V. Chaly, E. M. Paleolog, T. S. Kolesnikova, I. I. Tikhonov, E. V. Petratchenko, and N. N. Voitenok, "Neutrophil alpha-defensin human neutrophil peptide modulates cytokine production in human monocytes and adhesion molecule expression in endothelial cells," European Cytokine Network, vol. 11, no. 2, pp. 257266, 2000.

[44] J. W. Lillard Jr., P. N. Boyaka, O. Chertov, J. J. Oppenheim, and J. R. Mcghee, "Mechanisms for induction of acquired host immunity by neutrophil peptide defensins," Proceedings of the National Academy of Sciences of the United States of America, vol. 96, no. 2, pp. 651-656, 1999.

[45] Z. Zhao, Z. L. Mu, X. W. Liu et al., "Expressions of antimicrobial peptides LL-37, human $\beta$ defensin- 2 and -3 in the lesions of cutaneous tuberculosis and tuberculids," Chinese Medical Journal, vol. 129, no. 6, pp. 696-701, 2016.

[46] A. Pivarcsi, I. Nagy, A. Koreck et al., "Microbial compounds induce the expression of pro-inflammatory cytokines, chemokines and human $\beta$-defensin-2 in vaginal epithelial cells," Microbes and Infection, vol. 7, no. 9-10, pp. 1117-1127, 2005.

[47] K. S. Kobayashi, M. Chamaillard, Y. Ogura et al., "Nod2dependent regulation of innate and adaptive immunity in the intestinal tract," Science, vol. 307, no. 5710, pp. 731-734, 2005.

[48] J. I. Castañeda-Sánchez, B. E. García-Pérez, A. R. MuñozDuarte et al., "Defensin production by human limbo-corneal fibroblasts infected with mycobacteria," Pathogens, vol. 2, no. 1, pp. 13-32, 2013.

[49] R. H. van den Berg, M. C. Faber-Krol, S. van Wetering, P. S. Hiemstra, and M. R. Daha, "Inhibition of activation of the classical pathway of complement by human neutrophil defensins," Blood, vol. 92, no. 10, pp. 3898-3903, 1998.

[50] A. A.-R. Higazi, I. I. Barghouti, and R. Abu-Much, "Identification of an inhibitor of tissue-type plasminogen activatormediated fibrinolysis in human neutrophils. A role for defensin," The Journal of Biological Chemistry, vol. 270, no. 16, pp. 9472-9477, 1995.

[51] E. E. Fomicheva, I. Iu. Pivanovich, O. V. Shamova, and E. A. Nemirovich-Danchenko, "Glucocorticoid hormones in immunomodulating effect of defensins," Rossiiskii Fiziologicheskii Zhurnal Imeni I.M. Sechenova, vol. 88, no. 4, pp. 496-502, 2002.

[52] S. Sharma, I. Verma, and G. K. Khuller, "Biochemical interaction of human neutrophil peptide-1 with Mycobacterium tuberculosis $\mathrm{H}_{37} \mathrm{Ra}$," Archives of Microbiology, vol. 171, no. 5, pp. 338-342, 1999.

[53] W. Wang, A. M. Cole, T. Hong, A. J. Waring, and R. I. Lehrer, "Retrocyclin, an antiretroviral theta-defensin, is a lectin," The Journal of Immunology, vol. 170, no. 9, pp. 4708-4716, 2003.

[54] S. Sharma and G. Khuller, "DNA as the intracellular secondary target for antibacterial action of human neutrophil peptide-I against Mycobacterium tuberculosis $\mathrm{H}_{37} \mathrm{Ra}$," Current Microbiology, vol. 43, no. 1, pp. 74-76, 2001.

[55] J. F. Gera and A. Lichtenstein, "Human neutrophil peptide defensins induce single strand DNA breaks in target cells," Cellular Immunology, vol. 138, no. 1, pp. 108-120, 1991.
[56] M. T. Silva, M. N. Silva, and R. Appelberg, "Neutrophil-macrophage cooperation in the host defence against mycobacterial infections," Microbial Pathogenesis, vol. 6, no. 5, pp. 369-380, 1989.

[57] A. Peschel, R. W. Jack, M. Otto et al., "Staphylococcus aureus resistance to human defensins and evasion of neutrophil killing via the novel virulence factor MprF is based on modification of membrane lipids with 1-lysine," The Journal of Experimental Medicine, vol. 193, no. 9, pp. 1067-1076, 2001.

[58] S. I. Miller, W. S. Pulkkinen, M. E. Selsted, and J. J. Mekalanos, "Characterization of defensin resistance phenotypes associated with mutations in the phoP virulence regulon of Salmonella typhimurium," Infection and Immunity, vol. 58, no. 11, pp. 37063710, 1990.

[59] M. E. Selsted, D. Szklarek, and R. I. Lehrer, "Purification and antibacterial activity of antimicrobial peptides of rabbit granulocytes," Infection and Immunity, vol. 45, no. 1, pp. 150-154, 1984.

[60] S. Sharma, I. Verma, and G. K. Khuller, "Antibacterial activity of human neutrophil peptide-1 against Mycobacterium tuberculosis H37Rv: in vitro and ex vivo study," European Respiratory Journal, vol. 16, no. 1, pp. 112-117, 2000.

[61] A. K. Lichtenstein, T. Ganz, T.-M. Nguyen, M. E. Selsted, and R. I. Lehrer, "Mechanism of target cytolysis by peptide defensins. Target cell metabolic activities, possibly involving endocytosis, are crucial for expression of cytotoxicity," The Journal of Immunology, vol. 140, no. 8, pp. 2686-2694, 1988.

[62] A. Lichtenstein, "Mechanism of mammalian cell lysis mediated by peptide defensins. Evidence for an initial alteration of the plasma membrane," The Journal of Clinical Investigation, vol. 88, no. 1, pp. 93-100, 1991.

[63] S. van Watering, P. J. Sterk, K. F. Rabe, and P. S. Hiemstra, "Defensins: key players or bystanders in infection, injury, and repair in the lung?" Journal of Allergy and Clinical Immunology, vol. 104, no. 6, pp. 1131-1138, 1999.

[64] A. Lichtenstein, T. Ganz, M. E. Selsted, and R. I. Lehrer, "In vitro tumor cell cytolysis mediated by peptide defensins of human and rabbit granulocytes," Blood, vol. 68, no. 6, pp. 1407-1410, 1986.

[65] A. K. Lichtenstein, T. Ganz, M. E. Selsted, and R. I. Lehrer, "Synergistic cytolysis mediated by hydrogen peroxide combined with peptide defensins," Cellular Immunology, vol. 114, no. 1, pp. 104-116, 1988.

[66] J.-I. Ashitani, H. Mukae, T. Hiratsuka, M. Nakazato, K. Kumamoto, and S. Matsukura, "Plasma and BAL fluid concentrations of antimicrobial peptides in patients with Mycobacterium avium-intracellulare infection," Chest, vol. 119, no. 4, pp. 11311137, 2001.

[67] M. Jacobsen, D. Repsilber, A. Gutschmidt et al., "Candidate biomarkers for discrimination between infection and disease caused by Mycobacterium tuberculosis," Journal of Molecular Medicine, vol. 85, no. 6, pp. 613-621, 2007.

[68] S. Sharma, I. Verma, and G. K. Khuller, “Therapeutic potential of human neutrophil peptide 1 against experimental tuberculosis," Antimicrobial Agents and Chemotherapy, vol. 45, no. 2, pp. 639-640, 2001.

[69] K. Ogata, B. A. Linzer, R. I. Zuberi, T. Ganz, R. I. Lehrer, and A. Catanzaro, "Activity of defensins from human neutrophilic granulocytes against Mycobacterium avium-Mycobacterium intracellulare," Infection and Immunity, vol. 60, no. 11, pp. 47204725, 1992.

[70] Y. Miyakawa, P. Ratnakar, A. G. Rao et al., "In vitro activity of the antimicrobial peptides human and rabbit defensins and porcine 
leukocyte protegrin against Mycobacterium tuberculosis," Infection and Immunity, vol. 64, no. 3, pp. 926-932, 1996.

[71] B. Mathew and R. Nagaraj, "Antimicrobial activity of human $\alpha$-defensin 5 and its linear analogs: N-terminal fatty acylation results in enhanced antimicrobial activity of the linear analogs," Peptides, vol. 71, pp. 128-140, 2015.

[72] J. Kang, D. Zhao, Y. Lyu et al., "Antimycobacterial activity of Pichia pastoris-derived mature bovine neutrophil $\beta$-defensins 5," European Journal of Clinical Microbiology \& Infectious Diseases, vol. 33, no. 10, pp. 1823-1834, 2014.

[73] J.-J. Kang, Y. Lyu, D.-M. Zhao et al., "Antimicrobial activity of recombinant mature bovine neutrophil $\beta$-defensin 4 on mycobacterial infection," International Journal of Tuberculosis and Lung Disease, vol. 19, no. 6, pp. 711-716, 2015.

[74] K. O. Kisich, M. Higgins, G. Diamond, and L. Heifets, “Tumor necrosis factor alpha stimulates killing of Mycobacterium tuberculosis by human neutrophils," Infection and Immunity, vol. 70, no. 8, pp. 4591-4599, 2002.

[75] O. Froy, "Regulation of mammalian defensin expression by Tolllike receptor-dependent and independent signalling pathways," Cellular Microbiology, vol. 7, no. 10, pp. 1387-1397, 2005.

[76] P. Méndez-Samperio, "Role of antimicrobial peptides in host defense against mycobacterial infections," Peptides, vol. 29, no. 10, pp. 1836-1841, 2008.

[77] K. O. Kisich, L. Heifets, M. Higgins, and G. Diamond, "Antimycobacterial agent based on mRNA encoding human $\beta$-defensin 2 enables primary macrophages to restrict growth of Mycobacterium tuberculosis," Infection and Immunity, vol. 69, no. 4, pp. 2692-2699, 2001.

[78] L. Corrales-Garcia, E. Ortiz, J. Castañeda-Delgado, B. RivasSantiago, and G. Corzo, "Bacterial expression and antibiotic activities of recombinant variants of human $\beta$-defensins on pathogenic bacteria and M. tuberculosis," Protein Expression and Purification, vol. 89, no. 1, pp. 33-43, 2013.

[79] L. Fattorini, R. Gennaro, M. Zanetti et al., "In vitro activity of protegrin-1 and beta-defensin-1, alone and in combination with isoniazid, against Mycobacterium tuberculosis," Peptides, vol. 25, no. 7, pp. 1075-1077, 2004.

[80] B. E. García-Pérez, D. A. Villagómez-Palatto, J. I. CastañedaSánchez et al., "Innate response of human endothelial cells infected with mycobacteria," Immunobiology, vol. 216, no. 8, pp. 925-935, 2011.

[81] P. T. Liu, M. Schenk, V. P. Walker et al., "Convergence of IL$1 \beta$ and VDR activation pathways in human TLR2/1-induced antimicrobial responses," PLoS ONE, vol. 4, no. 6, Article ID e5810, 2009.

[82] C. E. Rivas-Santiago, B. Rivas-Santiago, D. A. León, J. Castañeda-Delgado, and R. Hernández Pando, "Induction of $\beta$ defensins by L-isoleucine as novel immunotherapy in experimental murine tuberculosis," Clinical and Experimental Immunology, vol. 164, no. 1, pp. 80-89, 2011.

[83] D. A. León, M. J. Zumárraga, R. J. Oropeza et al., "Mycobacterium bovis with different genotypes and from different hosts induce dissimilar immunopathological lesions in a mouse model of tuberculosis," Clinical \& Experimental Immunology, vol. 157, no. 1, pp. 139-147, 2009.

[84] B. Rivas-Santiago, E. Sada, V. Tsutsumi et al., “ $\beta$-defensin gene expression during the course of experimental tuberculosis infection," The Journal of Infectious Diseases, vol. 194, no. 5, pp. 697-701, 2006.

[85] A. R. Cervantes-Villagrana, R. Hernández-Pando, A. Biragyn et al., "Prime-boost BCG vaccination with DNA vaccines based in $\beta$-defensin- 2 and mycobacterial antigens ESAT6 or Ag85B improve protection in a tuberculosis experimental model," Vaccine, vol. 31, no. 4, pp. 676-684, 2013.

[86] O. Chertov, D. F. Michiel, L. Xu et al., "Identification of defensin1, defensin-2, and CAP37/azurocidin as T-cell chemoattractant proteins released from interleukin-8-stimulated neutrophils," The Journal of Biological Chemistry, vol. 271, no. 6, pp. 29352940, 1996.

[87] M. C. Territo, T. Ganz, M. E. Selsted, and R. Lehrer, "Monocytechemotactic activity of defensins from human neutrophils," The Journal of Clinical Investigation, vol. 84, no. 6, pp. 2017-2020, 1989.

[88] A. Kalita, I. Verma, and G. K. Khuller, "Role of human neutrophil peptide-1 as a possible adjunct to antituberculosis chemotherapy," Journal of Infectious Diseases, vol. 190, no. 8, pp. 14761480, 2004.

[89] V. Driss, F. Legrand, E. Hermann et al., "TLR2-dependent eosinophil interactions with mycobacteria: role of $\alpha$-defensins," Blood, vol. 113, no. 14, pp. 3235-3244, 2009.

[90] P. Méndez-Samperio, L. Alba, and A. Trejo, "Mycobacterium bovis-mediated induction of human $\beta$-defensin- 2 in epithelial cells is controlled by intracellular calcium and p38MAPK," Journal of Infection, vol. 54, no. 5, pp. 469-474, 2007.

[91] B.-D. Zhu, Y. Feng, N. Huang, Q. Wu, and B.-Y. Wang, "Mycobacterium bovis bacille Calmette-Guérin (BCG) enhances human $\beta$-defensin-1 gene transcription in human pulmonary gland epithelial cells," Acta Pharmacologica Sinica, vol. 24, no. 9, pp. 907-950, 2003.

[92] M. Henao-Tamayo, C. A. Shanley, D. Verma et al., "The efficacy of the BCG vaccine against newly emerging clinical strains of Mycobacterium tuberculosis," PLoS ONE, vol. 10, no. 9, Article ID e0136500, 2015.

[93] D. Tran, P. A. Tran, Y. Q. Tang, J. Yuan, T. Cole, and M. E. Selsted, "Homodimeric theta-defensins from rhesus macaque leukocytes: isolation, synthesis, antimicrobial activities, and bacterial binding properties of the cyclic peptides," The Journal of Biological Chemistry, vol. 277, no. 5, pp. 3079-3084, 2002.

[94] B. Yasin, W. Wang, M. Pang et al., " $\theta$ Defensins protect cells from infection by herpes simplex virus by inhibiting viral adhesion and entry," Journal of Virology, vol. 78, no. 10, pp. 5147-5156, 2004.

[95] I. Gonzalez-Curiel, V. Trujillo, A. Montoya-Rosales et al., "1,25Dihydroxyvitamin $\mathrm{D}_{3}$ induces LL-37 and HBD-2 production in keratinocytes from diabetic foot ulcers promoting wound healing: an in vitro model," PLoS ONE, vol. 9, no. 10, Article ID el11355, 2014. 


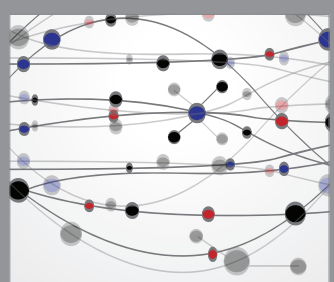

The Scientific World Journal
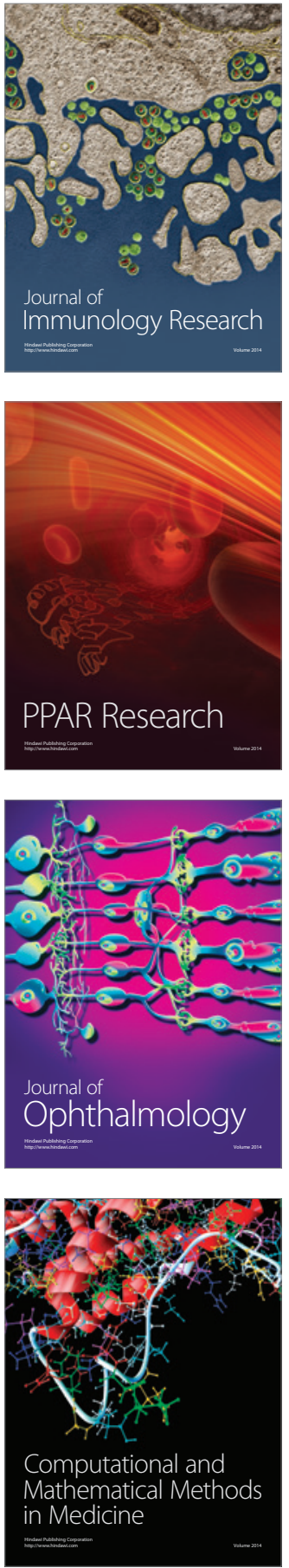

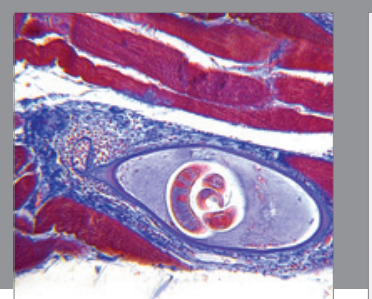

Gastroenterology Research and Practice

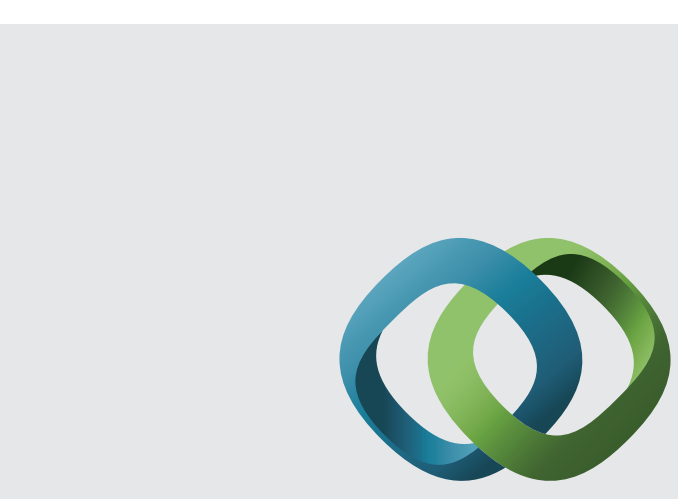

\section{Hindawi}

Submit your manuscripts at

http://www.hindawi.com
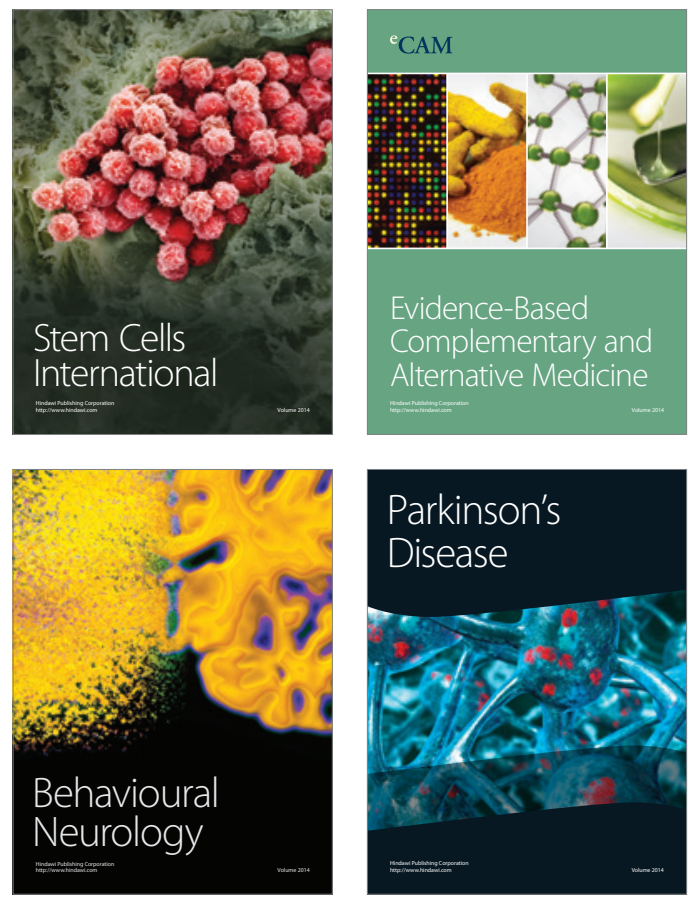
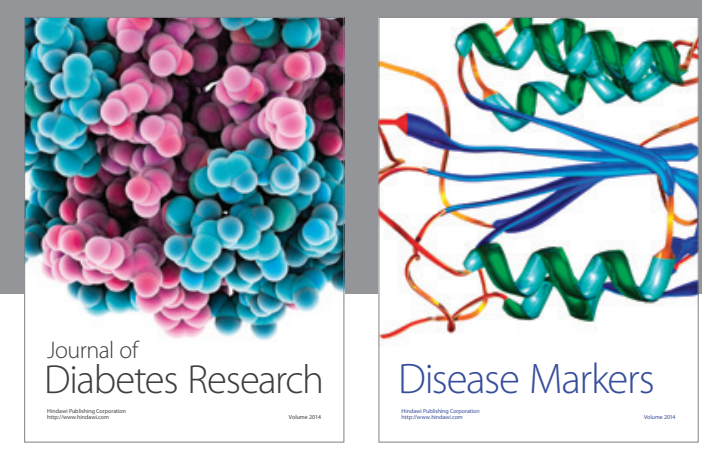

Disease Markers
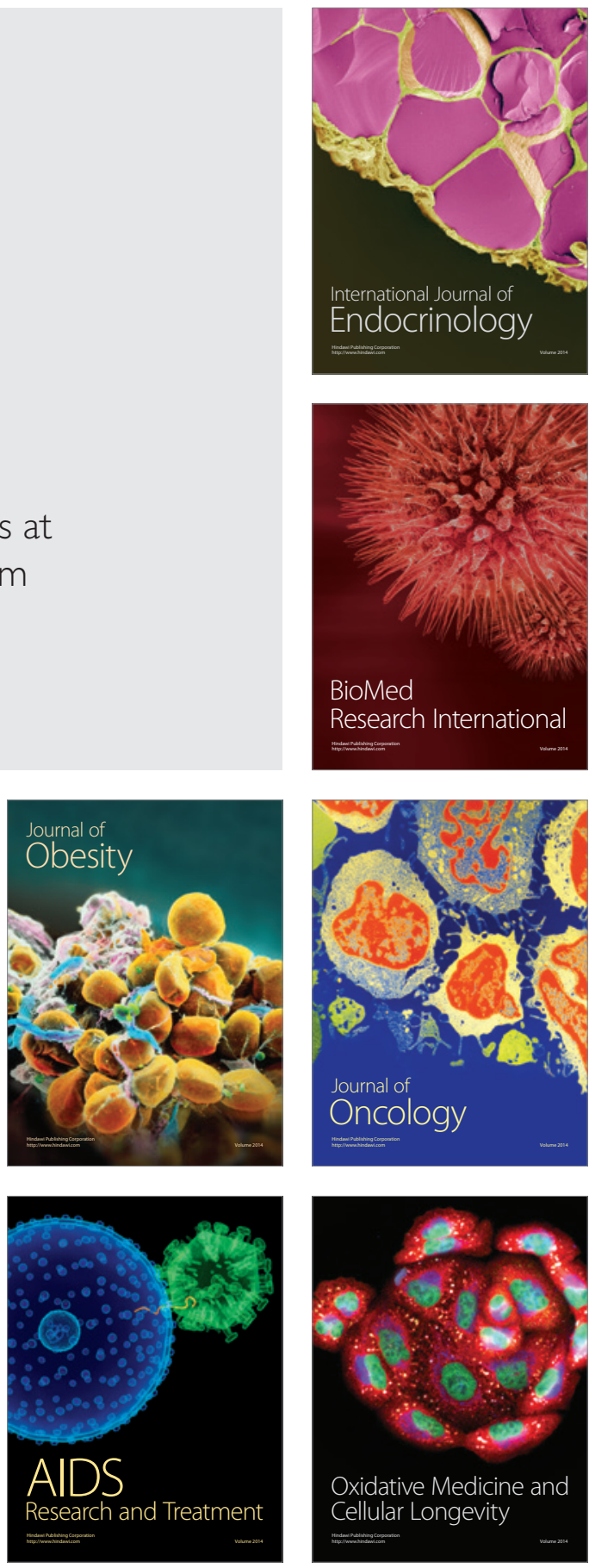